\title{
Anisotropic Outflows from LBVs and Ofpe/WN9 Stars
}

\author{
Antonella Nota ${ }^{1,2}$ \\ 1 Space Telescope Science Institute, 3700 San Martin Drive, Baltimore MD 21218, \\ USA \\ 2 Astrophysics Division, Space Science Department of the European Space Agency
}

\begin{abstract}
In the past decade, the increasing sophistication of the high resolution techniques from the ground, and the advent of HST have allowed a systematic study of the outflows around evolved massive stars. It has been established (Nota \& Clampin 1997) that most LBVs and Ofpe/WN9 stars are surrounded by associated circumstellar nebulae which have been ejected in some previous phase of their evolution. These nebulae are the fossil record of the interactions of previous winds and of the violent ejections in which the stars most likely have shed their outer layers. The study of the morphology, kinematics and chemical composition of the ejected material has allowed us to gain deep insight in the ejection and shaping mechanism.
\end{abstract}

\section{Are outflows isotropic?}

In this paper, I will review the observational evidence for the isotropy, or lack thereof, of outflows from luminous, evolved stars. I will be mainly concentrating on the large scale structures of nebulae around LBVs and Ofpe/WN9 stars, in the Milky Way and in the LMC. In order to establish whether the distribution of ejected material is isotropic or not, we study a) the morphology of the ionized and neutral material, from direct images in the optical and IR, b) the kinematics of the gas, which provides information on the nebular expansion motions.

In terms of morphology, LBV and Ofpe/WN9 associated nebulae always show the presence of a preferential symmetry axis. In Figure 1, we show three examples of the most "bipolar" nebulae, where such terminology is drawn from the PN classification. In such a structure, two expanding bubbles can be distinguished, aligned with a preferential axis, associated with a narrow waist. $\eta$ Carinae (Davidson et al. 1997; Schulte-Ladbeck et al. 1988b) is the textbook case of such a morphological type (Figure 1, left panel). The nebulae around HR Carinae (Hutsemékers \& Van Drom 1991a; Clampin et al. 1995; Weis et el. 1997; Nota et al. 1997; Hulbert et al. 1998) and the LBV candidate HD168625 (Hutsemékers et al. 1994; Nota et al. 1997; Robberto \& Herbst 1998) are also bipolar, although in these two cases the morphology is less visually striking. In the case of HR Carinae, the nebula is much older, and only the southern bubble is visible, and fragmented. As we will discuss later, 
the kinematic data confirms presence of a truly bipolar outflow. In the case of HD168625, the compact elliptical nebula in the center is likely to be the "waist" of the bipolar distribution. Such a waist is resolved into a horn shaped structure in mid-IR images obtained by Robberto \& Herbst (1998).
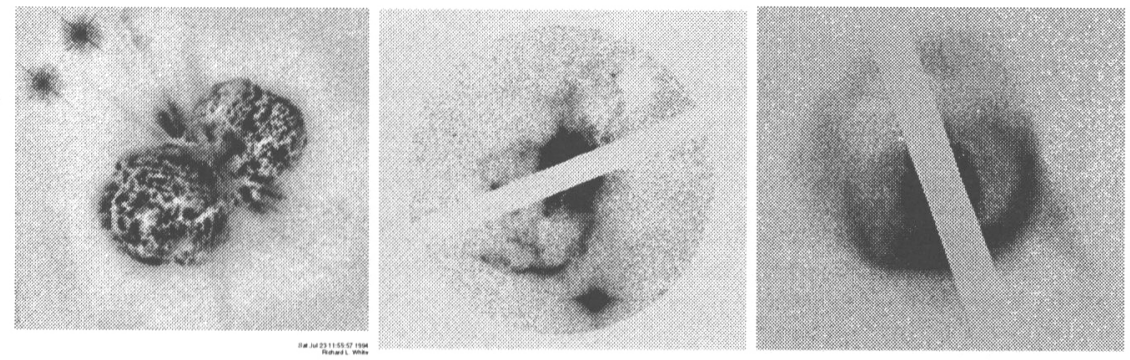

Fig. 1. Three examples of galactic extreme bipolar nebulae, in the light of $H \alpha: \eta$ Car (courtesy of R. White), HR Car, HD168625. In all images, North is up and East to the left.

Most of the remaining known nebulae display much milder asymmetries. AG Carinae (Smith et al. 1997; Trams et al. 1996; Schulte-Ladbeck et al. 1994; Leitherer et al. 1994), R127 (Walborn 1982; Stahl 1987; Clampin 1993; Smith et al. 1998) and Wra 751 (Hu et al. 1990; Hutsemékers \& van Drom 1991b; de Winter et al. 1992) are three representatives of the more conspicuos class of nebulae where a preferred axis can still be noted but the overall symmetry is elliptical (Figure 2).
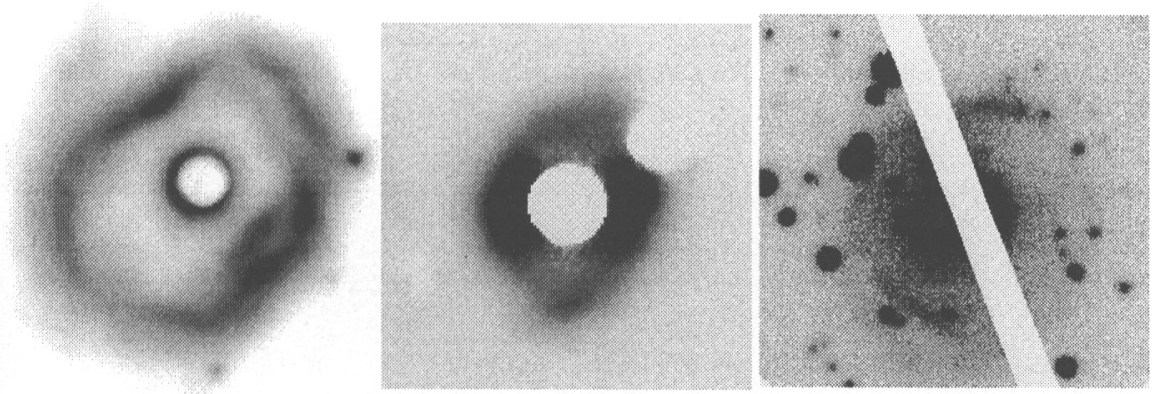

Fig. 2. Examples of elliptical nebulae in the light of $H \alpha$ : AG Carinae (galactic), R127 (LMC), Wra 751 (galactic). In all images North is up and East to the left. The images are not equally scaled. 
AG Carinae and R127 are remarkably similar: AG Carinae is galactic, $\simeq$ $36^{\prime \prime} \times 36^{\prime \prime}$ in size, while R127 is in LMC, and its apparent size is much smaller $\left(\simeq 8^{\prime \prime} \times 9^{\prime \prime}\right)$ (Figure 2: left and middle). Both nebulae are quite old (several tens of thousand years). In both cases it is possible to note the presence of a preferred axis (the major axis of the ellipse) and of two symmetrical regions of enhanced brightness, aligned with the minor axis.

In the currently accepted scenario that ejected nebulae are shaped by interacting winds (Nota et al. 1995; Frank, Balick \& Davidson 1995; GarciaSegura et al. 1996), the ratio of the density in the equatorial waist with respect to the polar region ultimately defines the observed morphology.

The fast blue supergiant wind is more effective at sweeping a region where the density is low, producing a more elliptical nebula when the density contrast is lower, and a more exteme bipolar nebula when the density contrast is higher. It is also interesting to note that all nebulae in Figure 1 are relatively young (100-5000 yrs) while the nebulae in Figure 2 are ten times older and more, indicating that evolution also plays a role in the formation of the currently observed morphologies.

Kinematical studies of these nebulae have subsequently confirmed what had been previously proposed on the basis of morphological considerations alone. For all known LBVs and Ofpe/WN9 associated nebulae, detailed kinematical studies can be found in the literature. In Figure 3, we show the kinematical maps for the two extreme cases: a bipolar outflow on the left panel (HR Carinae, Nota et al. 1997) and an expanding shell, on the right panel (S61, Pasquali et al. 1998). In both maps the radial velocity (reduced to the heliocentric system, in $\mathrm{km} \mathrm{s}^{-1}$ ) is plotted as a function of position with respect to the star (in arcseconds, east to the left, west to the right). In the case of S61, the data (filled dots) are modelled with a spherically symmetrical expansion law (empty circles). For most nebulae around LBVs and Ofpe/WN9 the kinematic maps fall in between these two extreme cases.
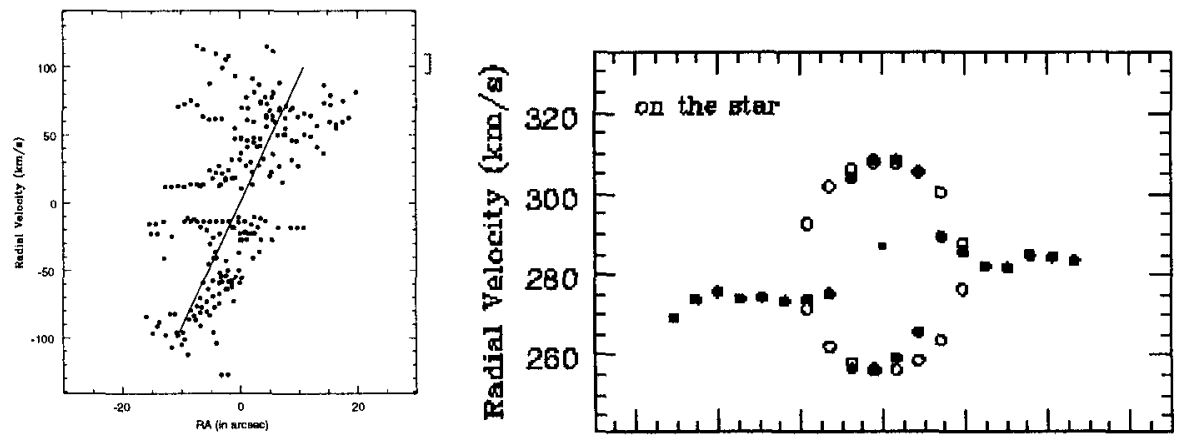

Fig. 3. Kinematic map for a) a bipolar nebula (HR Carinae) b) an expanding shell (S61) 
It is worth mentioning at this point the case of the WNL star Wra 124 which is surrounded by a large clumpy nebula, studied at high resolution both from the ground (Sirianni et al. 1998) and with HST (Grosdidier et al. 1998). On the basis of morphology alone it is difficult to establish the nebular structure: while the inner nebula seems to be dominated by a bipolar morphology (Sirianni et al. 1998), the large scale HST images reveal a miriad of fragmented clumps, arches and filament with no apparent overall structure (Grosdidier et al. 1998). However, the study of the kinematic properties help to discriminate at least two motions: an expanding shell and a superimposed bipolar outflow (Figure 4). Most likely, even more complex motions are present in the circumstellar environment of Wra 124.
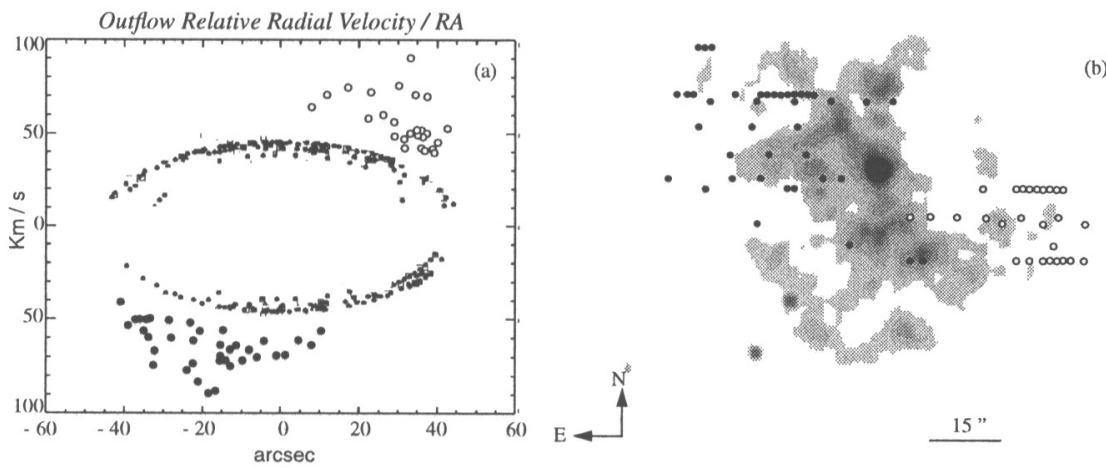

Fig. 4. Radial velocity map of the M1-67 nebula (left panel). The bipolar outflow is seen as the residual to the elliptical model of the expanding shell. The regions defining the bipolar outflow are marked on the $\mathrm{H} \alpha$ image of the nebula (right panel).

\subsection{The exception: P Cygni}

Although P Cygni is the prototypical Luminous Blue Variable, its surrounding nebula is certainly not the protypical LBV nebula: it is the only one to display a true spherical symmetry, and a highly nonhomogeneous distribution of clumps within a spherical envelope (Figure 5: left panel). The kinematic information (Nota et al. 1998) confirms the suggested scenario: in Figure 5 (right panel) we show the radial velocity map obtained with the long slit positioned at $8^{\prime \prime} \mathrm{N}$, intercepting the four brightest clumps in the northern hemisphere (B, D, F, H - Nota et al. 1998). While the overall profile is reminiscent of a spherically expanding shell, a more complex structure is dicernible in the line profile of the line $\mathrm{Fe}[\mathrm{II}] \lambda 4287$. The clumps appear to be both at 
the surface and inside the expanding shell. This finding is in agreement with Skinner et al. (1998) who, on the basis of radio data, concluded that 1) the circumstellar environment of $\mathrm{P}$ Cygni is very clumpy, 2) the clumps appear randomly distributed, and 3) in close proximity to the central star they are variable with timescale of days.
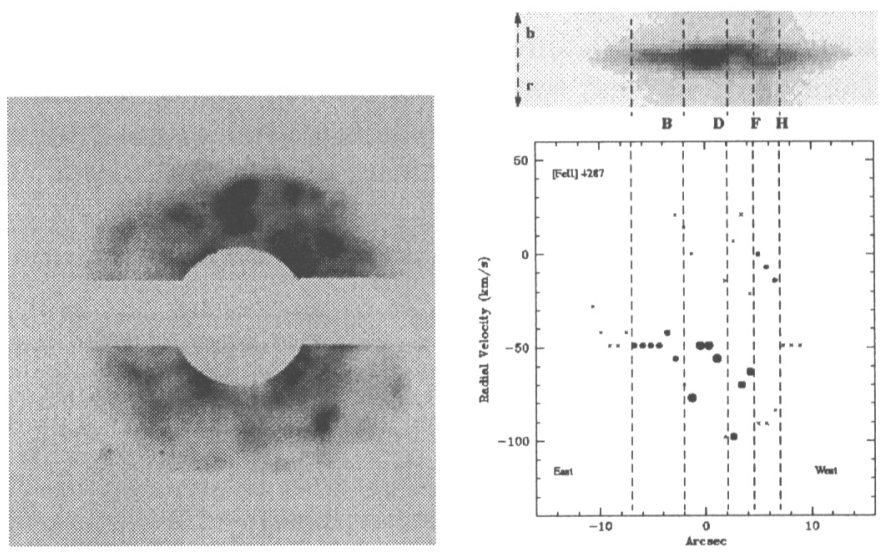

Fig. 5. Image of the P Cygni nebula in the light of $\mathrm{H} \alpha$ (left panel); a subset of the long slit image centered on the $\mathrm{Fe}[\mathrm{II}] \lambda 4287$ line (right panel). Note the complex structure of the nebular line, projected onto its corresponding kinematic profile.

\section{The connection between large scale structure and the stellar wind}

For a number of LBVs, a connection has been found between the large scale structure of the nebulae, up to a few parsecs from the star, and the structure of the stellar wind. In the case of AG Carinae, for example, Leitherer et al. (1994) noted that the stellar spectrum in the optical and the UV wavelengths is dominated by the effects of a massive stellar wind. They detected two wind components: a slow dense wind, where the bulk of the recombination radiation is emitted, and a faster, less dense wind, visible in the absorption components of the UV P Cygni profiles (with velocities up to $-1000 \mathrm{~km} \mathrm{~s}^{-1}$ ). At the same time, Schulte-Ladbeck et al. (1994) found very large variations of the polarization with time, along a preferred direction which is aligned with the major axis of the optical nebula (Figure 2, left panel). These two independent observations seem to indicate that the asymmetries noted in the ejected nebula are also present in the stellar wind, very close to the star.

Spectropolarimetry of the LMC LBV R127 in UBVRI, $\mathrm{H} \alpha$, [NII] has also shown large intrinsic continuum polarization (1-1.5\%) indicating both the 
presence of a substantial quantity of free electrons in the wind and a very large deviation from spherical symmetry very close to the star. Again, the direction of the polarization vector matches the nebular symmetry axis (SchulteLadbeck et al. 1993) from the optical images (Figure 2: middle panel).

Spectropolarimetry has proven to be a fundamental diagnostic tool also in cases where no asymmetries are present. Well before any images of the nebula around P Cygni had been obtained, Taylor et al. (1991) gathered optical spectropolarimetric measurements of $\mathrm{P}$ Cygni and found intrinsic polarization variable with time, on a short time scale, indicating that the scattering material lied close to the star. Moreover, they found a variation in the position angle of the polarization vector, indicating no preferential direction, and therefore they ruled out a spherically symmetric wind or an axisymmetric shell. When the first optical images of the nebula were obtained (Barlow et al. 1994; Nota et al. 1995), it was clear that spectropolarimetry had proven right: the nebula was composed of clumps, distributed within a spherically symmetrical envelope, withot any preferential axisymmetry. A few years later, Skinner et al. (1998) found that the radio emission close to the star is also highly inhomogeneous, with timescales of a few days. The connection between the stellar wind structure and the outer nebula holds for P Cygni, although in the context of a completely different morphological structure.

\section{A new result: the true $\mathrm{R} 143$ nebula}

R143 was identified by Parker (1993) as a LBV. R143 was believed to be surrounded by a circumstellar nebula, whose morphology was significantly different from all other observed LBV nebulae: three filaments (the "fingers) emerged from the star, ending in unresolved clumps (Figure 6, left panel): a very different morphology from all other known LBV nebulae.

From the discovery paper, it was not clear whether the nebula and the central star were physically associated: inspection of the surrounding region showed that R143 was located in an extended HII region, rich in gas and dust. Smith et al. (1998) therefore took long slit spectra to assess the nature of the nebula and found the three fingers not to be associated with the central star, both in terms of their kinematics and chemical composition, but to belong to the underlying HII region. However, they did find a compact nitrogen enriched region (Figure 6, right panel), in close proximity to the star, with a spatial extension of $2-4^{\prime \prime}$, which was subsequently resolved in HST/WFPC2 images as a small compact nebula most likely recently ejected by the central LBV (Schulte-Ladbeck et al. 1998a).

\section{Conclusions}

In this paper, I have reviewed the current observational evidence related to anisotropic outflows in LBVs and Ofpe/WN9 stars. Most luminous stars in 

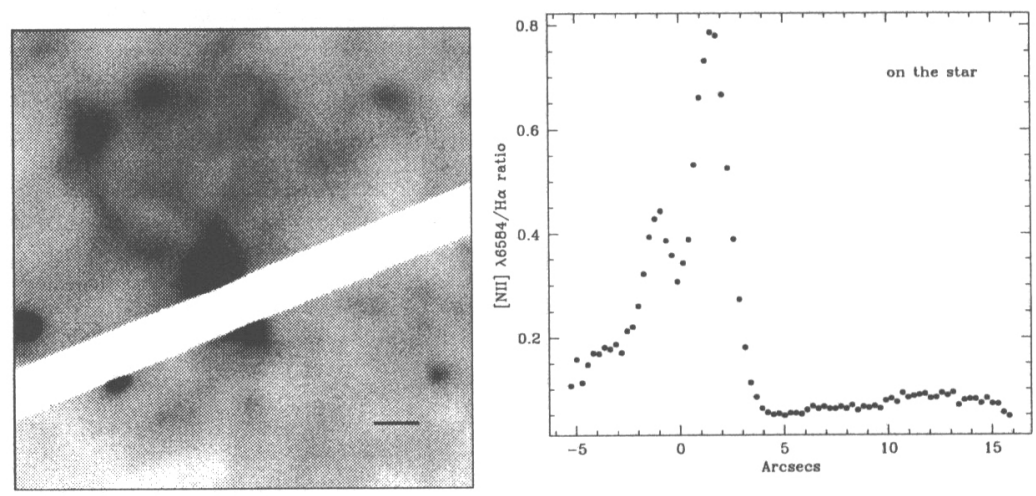

Fig. 6. $\mathrm{H} \alpha$ image of the R143 circumstellar region (left panel). The $\mathrm{N} / \mathrm{H}$ ratio is shown in the right panel as a function of position with respect to the star, indicating the presence of a nitrogen enriched region in close proximity to the central star.

these two categories are surrounded by spectacular circumstellar nebulae of ejected material. With the notable exception of one object, P Cygni, all nebulae display some level of axisymmetry, ranging from the true bipolar morphology of $\eta$ Carinae, to the more frequent elliptical nebulae. The kinematic information available confirms the morphological evidence that the outflows are not isotropic. In a few cases, using spectropolarimetric techniques, it has been possible to associate the structure of the stellar wind with the large scale structure of the ejected nebulae: in all these cases, a close analogy has been shown. In this context, ejected nebulae have shown to be truly the fossil record of the history of their previous winds. Hydrodynamical models have been able so far to reproduce qualitatively, quite successfully, the morphology of these nebulae. The time is now ripe to proceed to a quantitative application of the models, with the objective of reconstructing the nebular origin, and therefore, the evolutionary history of the central stars.

\section{Acknowledgements}

AN is grateful to friends and colleagues who have been integral part of the LBV nebulae project: Mark Clampin, Anna Pasquali, Regina SchulteLadbeck, Linda Smith.

\section{References}

Barlow, M.J., Drew,J.E., Meaburn,J. \& Massey, R.M. 1994, MNRAS 268, L29

Clampin, M., Nota, A., Golimowski, D.A., Leitherer, C. \& Durrance, S.T. 1993, ApJ 410, L35 
Clampin, M., Schulte-Ladbeck,R.E., Nota, A., Robberto, M., Paresce, F., \& Clayton, G. 1995 AJ 110, 251

Davidson, K., Ebbets, D., Johansson, S., Morse, J.A., Hamann, F.W., Balick, B., Humphreys, R., Weigelt, G. \& Frank, A. 1997, AJ 113, 335

de Winter, D., Peréz, M.R., Hu, J.H. \& Thé, P.S. 1992, A\&A 257, 632

Frank, A., Balick, B. \& Davidson, K. 1995, AJ 109, 178

Garcia-Segura, G., Langer, N. \& MacLow, M.,-M. 1996, A\&A 316, 133

Grosdidier, Y. Moffat, A.F.J., Joncas, G. \& Acker, A. 1998, ApJ 506, L127

Hu, J.H., de Winter, D., Thé, P.S. \& Peréz, M.R. 1990, A\&A 227, L17

Hulbert, S., Nota, A., Clampin, M., Leitherer, C., Pasquali, A., Langer, N. \& SchulteLadbeck, R.E. 1998, this conference

Hutsemékers, D. \& Van Drom,E. 1991a A\&A 248, 141

Hutsemékers, D. \& Van Drom, E. 1991b, A\&A 251, 620

Hutsemékers, D., Van Drom, E., Gosset, E. \& Melnick, J. 1994 A\&A 290, 906

Leitherer, C., Allen, R., Altner, B., Damineli, A., Drissen, L., Idiart, T., Lupie, O., Nota, A., Robert, C., Schmutz, W. \& Shore, S.N. 1994, ApJ 428, 292

Nota, A., Livio, M., Clampin, M. \& Schulte-Ladbeck, R.E. 1995, ApJ 448, 788

Nota, A., Pasquali, A., Clampin, M., Pollacco, D., Scuderi, S. \& Livio, M. 1996 ApJ 473,946

Nota, A. \& Clampin, M. 1997: Luminous Blue Variables: Massive Stars in Transition eds. A.Nota, H.J.L.M. Lamers (PASP), p.303

Nota, A., Smith,L.J., Pasquali, A., Clampin, M., \& Stroud, M. 1997 ApJ 486, 338

Nota, A.,

Clampin, M., Pasquali, A., Robberto, M., Pollacco, D., Ligori, S., Paresce, F. \& Staude, J. 1998 in preparation.

Parker, J.Wm., Clayton, G.C., Winge, C. \& Conti, P. 1993, ApJ 409, 770

Robberto, M., \& Herbst, T.M. 1998, ApJ 498, 400

Schulte-Ladbeck, R.E., Leitherer, C., Clayton, G.C., Robert, C., Meade, M.R., Drissen, L. Nota, A. \& Schmutz, W. 1993, ApJ 407, 723

Schulte-Ladbeck, R.E., Clayton, G.C., Hillier, D.J., Harries, T.J. \& Howarth, I.D. 1994, ApJ 429, 846

Schulte-Ladbeck, R.E., Pasquali, A., Clampin, M., Nota, A., Hillier, D.J.\& Lupie, O. 1998, this conference.

Sirianni, M., Nota, A., Pasquali, A. \& Clampin, M. 1998, A\&A 335, 1029

Skinner, C., Becker, R.H., White, R.L., Exter, K.M., Barlow, M.J. \& Davis, R.J. 1998, MNRAS 296, 669

Smith, L.J., Stroud, M.P., Esteban, C., Vilchez, J.M. 1997, MNRAS 290, 265

Smith, L.J., Nota, A., Pasquali, A., Leitherer, C., Clampin, M. \& Crowther, P.A. 1998 ApJ 503, 278

Stahl, O. 1987 A\&A 182, 229

Taylor, M., Nordsieck, K.H., Schulte-Ladbeck, R.E. \& Bjorkman, K.S. 1991 AJ 102, 1197

Trams, N.R., Waters, L.B.F.M. \& Voors, R.H.M. 1996, A\&A 315, L213

Walborn, N.R. 1982, ApJ 256, 452

Weis, K., Duschl, W.L., Bomans, D.J., Chu, Y.-H., \& Joner, M.D. 1997 A\&A 320, 568 


\section{Discussion}

K. Weis: For the sake of completeness I would like to comment that Weis et al. (1997, A\&A 320, 568; April 1997) already showed the bipolar structure of the nebula around HR Car before Nota et al. (1997, ApJ 486, 338; September 1997).

S. Shore: It is worth noting that for several LBVs the bolometric luminosity remains constant. This implies that the outflows are, if not spherical, at least optically thick right down near the star.

MWC 560 has not been mentioned at this meeting, but is well known to show a detached absorption line. Yes, it is a symbiotic and $L \sim 10^{3} L_{\odot}$, but don't hold that against it!

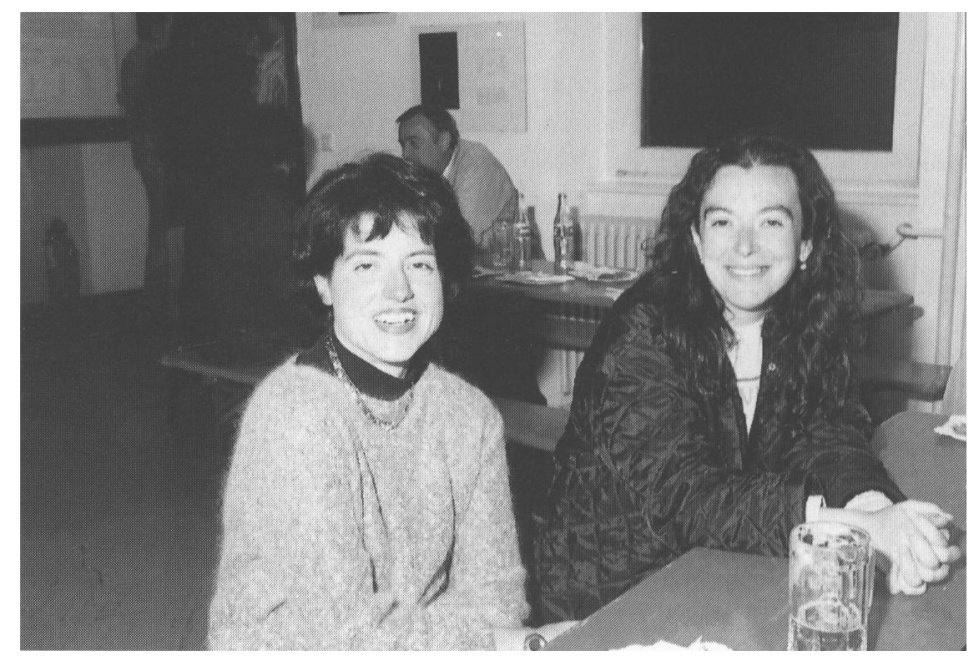

Antonella Nota and Anna Pasquali 